Portland State University

PDXScholar

$9-1-2005$

\title{
The Ultrasonic/Shear-Force Microscope: Integrating Ultrasonic Sensing into a Near-Field Scanning Optical Microscope
}

Andres H. La Rosa

Portland State University, andres@pdx.edu

Xiquan Cui

Portland State University

J. McCollum

Portland State University

Nan Li

Portland State University

Richard Nordstrom

Portland State University

Follow this and additional works at: https://pdxscholar.library.pdx.edu/phy_fac

Part of the Physics Commons

Let us know how access to this document benefits you.

\section{Citation Details}

La Rosa, A. A., Cui, X. X., McCollum, J. J., Li, N. N., \& Nordstrom, R. R. (2005). The ultrasonic/shear-force microscope: Integrating ultrasonic sensing into a near-field scanning optical microscope. Review Of Scientific Instruments, 76(9), 093707.

This Article is brought to you for free and open access. It has been accepted for inclusion in Physics Faculty Publications and Presentations by an authorized administrator of PDXScholar. Please contact us if we can make this document more accessible: pdxscholar@pdx.edu. 


\title{
The ultrasonic/shear-force microscope: Integrating ultrasonic sensing into a near-field scanning optical microscope
}

\author{
A. La Rosa, a) X. Cui, J. McCollum, and N. Li \\ Department of Physics, Portland State University, P.O. Box 751, Portland, Oregon 97207 \\ R. Nordstrom \\ Mechanical and Materials Engineering Department, Portland State University, P.O. Box 751, \\ Portland, Oregon 97207
}

(Received 22 April 2005; accepted 13 August 2005; published online 15 September 2005)

\begin{abstract}
An ultrasonic transducer is incorporated into a near-field scanning optical microscope (NSOM) to augment its versatility to characterize the properties of layers adsorbed to a sample's surface. Working under typical NSOM operation conditions, the ultrasonic transducer-attached underneath the sample-demonstrates sufficient sensitivity to monitor the waves generated by the tapered NSOM probe that oscillates in the proximity of, and parallel to, the sample's top surface. This capability makes the newly integrated ultrasonic/shear-force microscope a valuable diagnostic tool in the study of sliding friction and surface phenomena in general. Here, it is used to concurrently and independently monitor the effects that probe-sample interactions exert on the probe (that is attached to a piezoelectric tuning fork) and on the sample (that is attached to the ultrasonic transducer). The signal from the tuning fork (TF) constitutes the so called "shear-force" signal, widely used in NSOM as a feedback to control the probe's vertical position but whose working mechanism is not yet well understood. Tests involving repeated vertical $z$ motion of the probe towards and away from the sample's surface reveal that the TF and ultrasonic (US) signals have distinct $z$ dependence. Additionally, where the TF signal showed abrupt changes during the approach, the US changed accordingly. A shift in the probe's resonance frequency that depends on the probe-sample distance is also observed through both the TF and the US responses. Within the sensitivity of the apparatus, ultrasonic signals were detected only at probe-sample distances where the probe's resonance frequency had shifted significantly. These measured signals are consistent with a probe entering and leaving a viscoelastic fluid-like film above the sample. The film acts as the medium where waves are generated and coupled to the ultrasonic sensor located beneath the sample. To our knowledge, this is the first reported use of ultrasonic detection for detailed monitoring of the distance dependence of probe-sample interactions, and provides direct evidence of sound as an energy dissipation channel in wear-free friction. This newly integrated ultrasonic/shear-force microscope, which can be implemented with any functionalized proximal probe (including aperture and apertureless NSOM), can become a valuable metrology tool in surface science and technology. () 2005 American Institute of Physics. [DOI: 10.1063/1.2052649]
\end{abstract}

\section{INTRODUCTION}

Near-field scanning optical microscopy (NSOM) comprises techniques that surpass the diffraction-limited lateral resolution encountered in conventional (far-field) optical microscopy. ${ }^{1,2}$ NSOM can be implemented in either apertureless ${ }^{3}$ or aperture ${ }^{4}$ modalities. In the latter case (preferable for applications where restricted light exposure is required) NSOM makes the mesoscopic domain accessible to optical metrology by (1) illuminating the sample through a subwavelength-sized aperture (less than $100 \mathrm{~nm}$ ), and (2) by laterally scanning the aperture while keeping its vertical position very close to the sample surface $(\sim 10 \mathrm{~nm})$. Typically, an aluminum-coated tapered fiber with an aperture at the apex serves as a NSOM probe. Critical to the NSOM operation is the control of the tip-sample distance in order to (a)

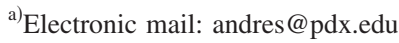

keep the excitation source in the (subwavelength) near-field region, (b) prevent the probe's crashing and destroying its aperture during scanning, and (c) avoid artifacts in the nearfield image (due to the sensitivity of the near-field optical signal to nanometer variations in the tip-sample distance).

Currently, a "shear force" (SF) measurement, which can be implemented by either optical ${ }^{4,5}$ or electrical ${ }^{6,7}$ means, is employed in NSOM to control the probe's vertical position. In the latter implementation, the piezoelectric resonance response of a mechanically or electrically driven quartz tuning fork (TF), with the NSOM probe attached to one of the tines, serves to monitor the probe's amplitude of oscillation. Within the shear-force interaction range there is a monotonic dependence of the SF with the tip-sample distance: the closer the tip to the sample, the smaller the oscillation amplitude. Practically, this relation is sufficient to implement a probe-sample distance control. However, the mechanism that gives rise to 
the SF measurement is not well understood, which creates uncertainty as to whether or not probe-sample distances are really being measured.

In the beginning, it was assumed that the SF measurement resulted from damping forces acting on the probe by the fluid-like film that is typically found adsorbed on almost any surface at ambient conditions. However, the SF, it turns out, is not a purely damping process as its name may suggest. Depending on the probe-sample distance, a decrease in amplitude is accompanied by an increase in the probe's resonance frequency, as is seen in the results provided here as well as elsewhere. Such an increase in the resonance frequency indicates that other forces must be acting on the tip, producing the net result of increasing the probe's effective spring constant. The participating forces may be of multiple origins and/or perhaps acting at different distances. ${ }^{8}$ They may include electrostatic, capillary, Van der Waals, surface tension, viscosity, ${ }^{9}$ mechanical nonlinear bending forces, ${ }^{10}$ intermittent contact between the tip and sample's surface, ${ }^{11}$ or intermittent contact between the tip and adlayers (instead of the solid sample's surface). ${ }^{12}$ Further, some of these interactions may cause the same effect under different working conditions; although some proposed mechanisms clearly require an atmosphere, shear-forces have even been measured under vacuum conditions. ${ }^{13}$ Currently there is not a consensus despite many efforts to understand the SF mechanism. Still, a correct understanding of the tip-sample interaction is needed to ensure reliable interpretations of high-resolution near-field imaging. The development of new metrology tools that can help clarify this problem would be highly desirable.

This article describes an alternative and complementary method to monitor probe-sample interactions based on ultrasonic detection of the waves generated by a laterally oscillating probe as it interacts with the specimen. The specimen in this case includes both the sample and its adsorbed fluidlike film. The new method differs from other ultrasonic techniques where the probe dithers perpendicular to the sample, ${ }^{14}$ or is kept stationary while an ultrasonic wave is applied to the sample. ${ }^{15}$ In the ultrasonic/shear-force microscope setup described here, the signal from the TF (to which the probe is attached) senses the effects that the probe-specimen interactions have on the probe while the ultrasonic sensor monitors the effects on the specimen. Thus, this method enriches the information available to interpret the intervening probespecimen interactions. Both signals are measured concurrently and appear sufficiently different so that they can be contrasted to give more insights than either one by itself. The virtue of the combined ultrasonic/shear-force system is that it operates at typical NSOM operation conditions (not requiring probe's oscillations exceedingly large).

\section{EXPERIMENTAL SETUP FOR THE MEASUREMENT OF THE ULTRASONIC AND SHEAR-FORCE SIGNALS}

Figure 1 shows a schematic of the three main sections of the ultrasonic/shear-force microscope's experimental setup: the probe and the specimen (a glass cover slide), the shear force detection system (displayed in the upper side), and the ultrasonic sensory arrangement (displayed in the lower side).

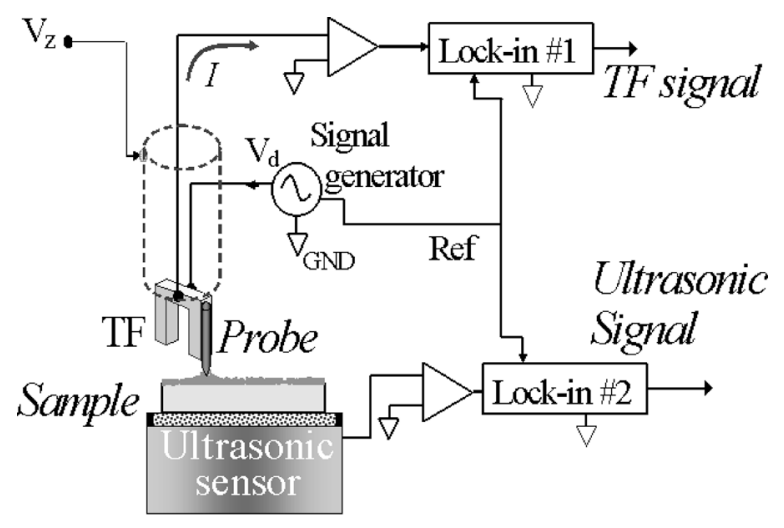

FIG. 1. Experimental setup for simultaneous measurement of the tuning fork and ultrasonic signals.

Considering that the probe-specimen interaction may depend on different parameters, including tip geometry, chemical nature, metal coating quality, etc., an uncoated tapered fiber was used in order to minimize the intervening interactions. A chemically etched ${ }^{16,17}$ fiber (3M FS-SC-6324), having an apex radius of $\sim 100 \mathrm{~nm}$ and a tapered region of approximately $200 \mu \mathrm{m}$ in length, was attached to one of the tines of a commercially available quartz crystal tuning fork.

Three variables are under experimental control: the tuning fork's driving voltage, the driving frequency, and the probe's vertical position. Two signal responses are monitored-the TF and the ultrasonic. The signal generator driving the TF supplies a programmable frequency and a programmable alternating current voltage $V_{d}$, (set to $10 \mathrm{mV}_{\mathrm{rms}}$ ), which causes the TFs tines to oscillate opposite to each other. The third experimental control is for the fine approach of the TF. To that effect, a piezoelectric tube (from EBL Products, Inc. EBL No. 3, 0.5-mm-thick, 50-mm-long, $20 \mathrm{~mm}$ outside diameter, and shown in dashed lines in Fig. 1 ) was attached to the TF. Changes in the voltage $V_{z}$, applied to the piezoelectric tube's inner electrode, correspond to changes in the tip's vertical position.

The so-called shear force signal is monitored by the output of the lock-in amplifier No.1 (SRS 850) that synchronously detects the root-mean-square (rms) amplitude of the alternating current generated by the electrically driven TF. At $V_{d}=10 \mathrm{mV}_{\text {rms }}$ and at a probe-sample distance approximately greater than $20 \mu \mathrm{m}$, far from operation proximity, the tuning fork displays a sharp resonance peak of $f_{0}=32679 \mathrm{~Hz}$ [as seen in Fig. 2(a)], and a quality factor $Q=(3)^{1 / 2} f_{0} /(\Delta f)_{1 / 2}$ $\sim 1800$. The peak current at the resonance frequency is $I_{\max }=4.4 \times 10^{-8} \mathrm{~A} \mathrm{rms}$ (corresponding to the tine's maximum oscillation amplitude used in this experiment), and at the plateau the current is $I_{\max }=3.3 \times 10^{-8}$ A rms (the tine's minimum oscillation amplitude). These two levels of current served as a guide to avoid crashing the tip when moving the probe toward the sample surface, and were used as the effective TF signal range in this experiment.

An ultrasonic transducer (SE40-Q, from Dunegan Engineering Consultants, Inc.) was chosen with a resonance frequency response near $40 \mathrm{kHz}$ and with sufficient high sensitivity near $32 \mathrm{kHz}(1 \mathrm{~V} / \mathrm{nm}$ sensitivity across its full active 10-15 mm diameter surface). Other ultrasonic transducers 


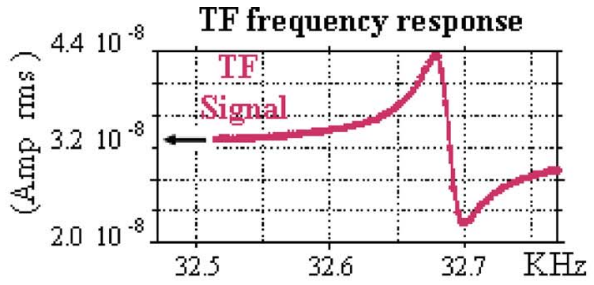

a)

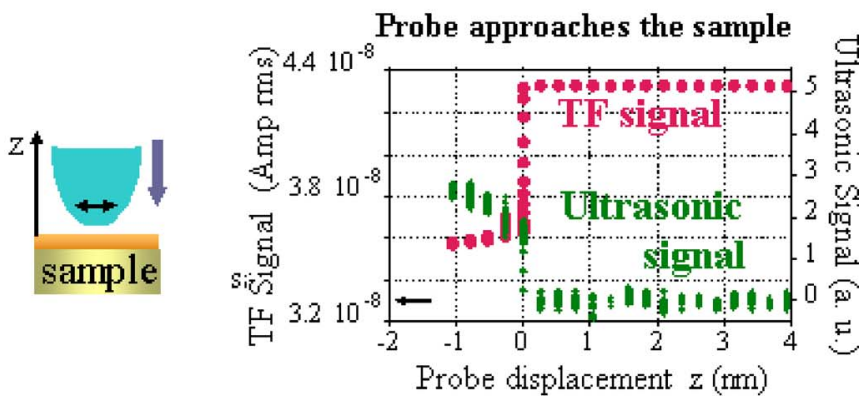

b)
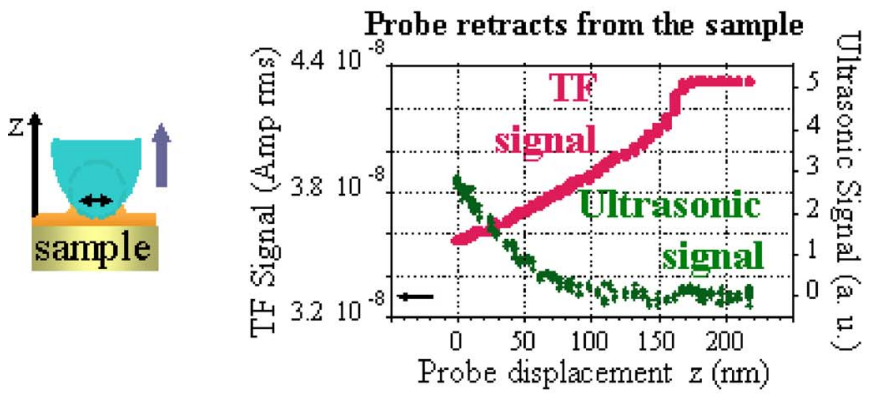

c)

FIG. 2. (a) Probe's frequency response taken at a probe-sample distance approximately greater than $20 \mu \mathrm{m}$. (b) Simultaneous monitoring of the tuning fork response and the ultrasonic signals as the probe approaches and (c) retracts, from the sample; taken at the driving frequency of $32679 \mathrm{~Hz}$.

were used successfully, but the SE40-Q gave the best signalnoise ratio, presumably because its bandwidth response matched closest with the frequency range used in this experiment. A thin layer of vacuum grease was applied between the glass cover slide and the surface of the ultrasound transducer's ceramic wear plate to ensure a good coupling. The ultrasonic transduce's signal was amplified and fed into a lock-in amplifier No.2 (a SR510 system with only one phase detection) also referenced to the TFs driving frequency. The output of this lock-in is referred to here as the "ultrasonicsignal."

\section{EXPERIMENTAL RESULTS}

In a typical operation, the probe's apex is positioned first at $\sim 20 \mu \mathrm{m}$ from the surface and set to vibrate at its resonance frequency using a driving voltage of $10 \mathrm{mV}_{\mathrm{rms}}$. Concurrently, the phase of the lock-in No.2 is set to provide a zero output reading. These constitute the initial condition settings for the experiment. The probe's estimated amplitude of oscillation and the equivalent driving force are $\sim 4 \mathrm{~nm}$ rms and $60 \mathrm{nN}$, respectively. ${ }^{18,19}$

\section{A. Approaching curves and correlation between the SF and US signals}

Figures 2(b) and 2(c), where $z$ is the relative vertical position of the probe, show how the TF and ultrasonic (US) signals vary as the probe approaches and retracts from the surface. Notice in the approaching curve [going from right to left in Fig. 2(b)] that the sudden decrease in the TF signal at $z=0$ coincides with the sudden increase in the US signal. This demonstrates the existing correlation between these two signals. When the probe is retracted [from left to right in Fig. $2(c)]$ both signals change more gradually, although (in this particular experiment) the almost linear behavior in the SF contrasts with the somewhat more quadratic variation of the ultrasonic one.

Notice in Fig. 2(c) that at $z>80 \mathrm{~nm}$ no ultrasonic signal is detected (within the current sensitivity of our apparatus) even though the TF has still not reached its maximum value. As we will show later, no major shift in the probe's resonance frequency is observed in this outer region either. Thus, the ultrasonic detection capability of the new microscope allows for identifying two dissimilar probe-specimen interaction regions that are not directly revealed by the TF signal alone. Finally, in the last part of the retraction trace, a sudden jump in the TF signal towards its maximum value is observed but without any change in the ultrasonic signal.

\section{B. Resonance frequency shift}

Figures 3(a) and 3(b) show frequency sweeps for both the shear-force and the ultrasonic signals, taken at the probe's different vertical positions $z$. These spectra were recorded while retracting the probe from the sample. The traces with open circles correspond to a position of the probe closer to the surface whereas the traces with open triangles were recorded when the probe's tip was away from the surface. The recording of each trace took $40 \mathrm{~s}$.

Notice that for decreasing values of $z$, while the peak values of the TF and ultrasonic signals decrease and increase, respectively, both signals sense an increase in the probe's resonance frequency (a consistent trend observed when using different samples and probes). This tendency continues until the TFs resonance peak approaches a minimum [as emphasized in Fig. 3(c) by the thick solid line joining the peaks of the different spectra]. In the insets of Fig. 3, the $z=0$ has arbitrarily been chosen at this minimum, since it appears that the tip of the probe is making a hard (solid-solid) contact with the sample at that location. The minimum, however, is not that sharp. For even smaller values of $z$, the peak amplitude of the TF instead increases (in this particular experiment); the peak of the US signal, on the other hand, keeps invariably increasing as $z$ decreases. (Such simultaneous increase of both the TF and ultrasonic signals at very short probe-sample distances is not universal and rather depends on the samples and probes used, probably owing to the different nature of the involved interactions in each case.)

One of the most compelling results reported here is that the ultrasonic signal is clearly distinguishable from the noise level [see the "filled squares" curve in Fig. 3(b)] only when a significant change in the resonance frequency exists [ap- 
proximately $5 \mathrm{~Hz}$ for the 'filled squares' curve, as seen in the inset of Fig. 3(c)]. That is, the greater the frequency shift, the greater the ultrasonic signal.

\section{DISCUSSION}

\section{A. On the existence of a fluid-like layer and the generation of the ultrasound waves}

The sudden jump displayed by the TF signal at the end of the retraction curve in Fig. 2(c), resembles the typical fingerprint of a stylus detaching from an adsorbed layer, similar to the retraction curves frequently seen in atomic force microscopy experiments. This sudden change in the TFs signal during the retraction, together with the abrupt change in both the US and TF signals during the approach [as seen in Fig. 2(b)], are indicative of a probe entering and leaving the boundary of a fluid-like layer adsorbed to the sample's surface. Incidentally, the existence of such a layer, particularly at ambient conditions, has been proposed earlier and it is widely accepted. ${ }^{20,21}$ Its origin relies on the hydrophilic character of the sample (like the glass substrate used in this experiment) to attract water molecules from the surrounding humid air, which leads to the formation of a layer several nanometers thick. Other hydrocarbon molecules might also be present in this layer. Based on the experimental data presented, this manuscript highlights the role played by this layer on the generation of the ultrasonic signal. In effect, the lack of ultrasound signal before the probe encounters the film [Fig. 2(b)] suggests that the immersion of the probe into the fluid-like film creates the waves, which subsequently propagate towards the ultrasonic sensor located beneath the sample.

Notice also in Fig. 2 that after the tip encountered the air/fluid-film boundary (presumably followed by the formation of a fluid meniscus around the probe), the probe was advanced no more than one nanometer before being immediately retracted. Also, at no point did the TF signal reach its nominal minimum level of $3.3 \times 10^{-8} \mathrm{~A}$ [the zero amplitude of oscillation level suggested by the spectrum in Fig. 2(a)]. It can be stated with certainty, then, that during the retraction, no solid-solid contact between the tip and the sample's surface occurred; still, an ultrasonic signal clearly above the noise level was detected. This result confirms again the role played by the fluid-like film in the generation of the ultrasonic signal; that is to say that ultrasonic waves are generated even when the probe is not tapping on the hard sample's surface, providing a direct demonstration of sound as one of the channels through which the probe dissipates its energy.

\section{B. The role of capillary forces and liquid bridges}

Although more gradual transitions from high to low levels in the TF signal are reported frequently, the abrupt change in the TF signal displayed in Fig. 2(b) suggests that capillary forces can have an influential role in the probesample interactions. In effect, due to the presence of condensed water films on the sample and on the probe, when the tip is placed close enough to the sample's surface a sudden formation of a liquid meniscus bridge can occur. The origin of this liquid bridge is either spontaneous capillary conden-
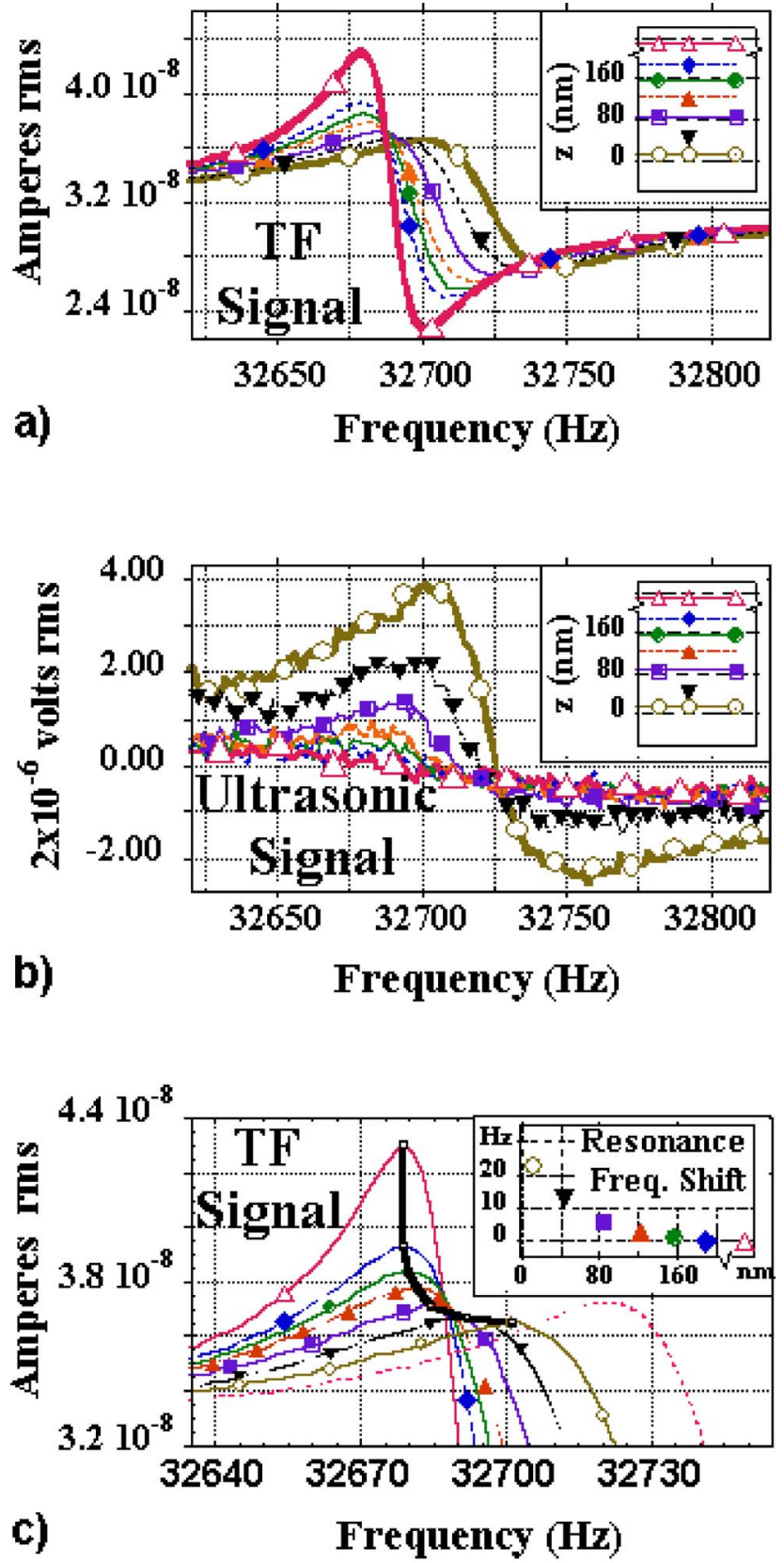

FIG. 3. (a) TF signal and (b) ultrasonic signal as a function of the driving frequency. Curves at different probe-sample distances are shown. (c) Zoom-in of the same data shown in (a), this time with a thick line joining the peaks of each frequency trace. The inset shows the shift in the resonance frequency as a function of the probe-sample distance. The $z=0$ reference is chosen at the point where the thick line reaches a minimum, which applies to the inset scales in (a) and (b). The dashed lines correspond to $z<0$.

sation or the result of a direct dipping of the tip into an already existent water film. ${ }^{22}$ The resulting force on the probe due to the bridge formation could be substantial. In the case of capillary condensation, for example, the attractive vertical force is $\sim 45 \mathrm{nN},{ }^{23,24}$ which is of the same order of magnitude of the $60 \mathrm{nN}$ force driving the probe. But, since the probe and the sample are firmly held by the microscope stage, this force is balanced. The forced lateral motion of the tip, however, changes the dynamics. Visualizing a liquid bridge with one side attached to the surface and the other to the tip, a horizontal displacement of the tip, $\Delta x$, would pro- 
duce a tilt and elongation of the bridge, with the consequent restoring horizontal component force $F_{x}$ acting on the probe, opposite to the tip's displacement. This force turns out to be $z$ dependent (for a bridge $10 \mathrm{~nm}$ long, $z=10 \mathrm{~nm}$, and a displacement $\left.\Delta x=4 \mathrm{~nm}, F_{x} \sim 45 \mathrm{nN} \times \Delta x / z \sim 15 \mathrm{nN}\right)$ and can be partially responsible for the changes in the probe's oscillation amplitude. It can contribute to the shift of the probe's resonance frequency as well $\left(\Delta f_{\text {res }}=3 \mathrm{~Hz}\right.$ at $\left.z=10 \mathrm{~nm}\right){ }^{25}$ The large retraction curve observed in Fig. 2(c) also supports the argument about the formation of a sticky bridge. In this view, as the probe retracts from the surface, the meniscus bridge deforms and gradually loosens. This makes the probe less effective to shake the layer, causing the ultrasonic signal to decrease while the TF signal increases. The last jump of the TF signal represents the reminiscence of surface tension forces, too weak to efficiently generate sound waves at the moment the bridge detaches from the tip or the surface.

\section{The role of viscosity}

Due to the complexity of the problem we cannot rule out, of course, the influence of other types of interactions, in particular the ones that have found relevance in other experiments involving shear force measurements. That is the case of viscosity. ${ }^{9,13,22}$ For example, a decrease in the probe's oscillation amplitude (the shear force signal) with decreasing probe-sample distance has been observed even under vacuum conditions. ${ }^{13}$ Evaluation of the dampening effects from electromagnetic as well as electrostatic-mediated interactions, however, turns out to be many orders of magnitude lower than the dampening effect measured in that experiment. The buildup of material in the tip-sample gap (even at vacuum conditions) was left as a possible source of the damping effects. ${ }^{13}$ In our case, the concurrent decrease in the probe's oscillation amplitude and the widening of the resonance curves as $z$ decreases (as seen in Fig. 3), also indicates an energy damping effect. Accordingly, we also explore an interpretation of our experimental results based on the viscous effect of the adsorbed layer.

More precisely, based on the results displayed in Fig. 2(c), we will have to consider a distance-dependent viscosity, $\eta(z)$, in order to explain the fact that the ultrasonic signal vanishes at $z>80 \mathrm{~nm}$ while the probe is still oscillating (with even increasing amplitudes as $z$ increases). Indeed, $a$ priori one would have expected to detect a stronger ultrasound signal at the probe's large oscillation amplitudes. However, the opposite occurs in this case: when the tip retracts a bit, the amplitude of the oscillation increases, but the ultrasonic signal decreases. This result suggests that the onset of the ultrasonic signal is not caused by the mechanical motion per se of the molecules in the layer, but instead by the ability of the laterally oscillating layer to transmit mechanical momentum in the transverse direction towards the ultrasonic sensor (located underneath the sample). This requirement is nothing but an invocation to the role that viscosity might play here. ${ }^{26} \mathrm{~A}$ viscosity that increases at shorter probe-sample distances would be compatible with our results: closer to the sample, the ultrasonic signal consistently strengthens due to a more efficient transfer of momentum; further away from the sample, it weakens.
Viscosity, however, has been one of the least suspected mechanisms involved in probe-sample interactions, ${ }^{8}$ although such claims are frequently based on calculations that ascribe bulk-values viscosity to the fluid-like film. As a reference, the force on a sphere of $50 \mathrm{~nm}$ in radius moving at speed $v \sim 5 \times 10^{-4} \mathrm{~m} / \mathrm{s}$ through liquid water would be $F_{\text {vis }}=6 \pi \eta_{\text {bulk }} R v=\sim 5 \times 10^{-4} \mathrm{nN}^{27}$ At the nanometer scale $(z<10 \mathrm{~nm})$, on the other hand, it is known that physical systems respond differently due to the ordering and sharp decrease in mobility of the constituent molecules when confined to small regions. When the film is a few monolayers thick, the effects are even more dramatic and their corresponding dynamic behavior cannot be understood by simple extrapolation from bulk properties. ${ }^{28}$ Further, some experimental results involving a gradual decrease of a fluid film's confined region have been interpreted as first-order phase transitions of the film from fluid to solid, ${ }^{29}$ although such experimental observations have proven to be very sensitive to surface quality. ${ }^{30}$ A gradual transition of the film from liquid to a glassy state has been suggested instead. ${ }^{31}$ (Incidentally, the ultrasonic/shear-force microscope introduced here can be a very useful alternative metrology tool helping to clarify such findings; a drastic change in the film's elastic properties should be reflected in a corresponding drastic change in the ultrasonic signal.) It appears, then, that contrary to predictions based on bulk properties calculations, viscosity may play a major role in modifying surface interactions at short (less than $10 \mathrm{~nm}$ ) probe-sample distances. It is possible that the viscous effects, although present, are not clearly differentiated in our experiments due to the capillary effects described above. Experiments with the tip immersed in bulk liquid (currently underway) should better clarify the effects of viscosity at distances lower than $10 \mathrm{~nm}$.

\section{On the role of viscoelasticity and the generation of sound}

Figure 3 provides additional insights about the role that the fluid-like film plays in the probe-specimen interactions. The fact that the ultrasonic signal becomes clearly noticeable only when the probe's resonance frequency shifts significantly constitutes one of the most compelling experimental results presented here. The filled squares trace in Fig. 3(b), which clearly stands above the noise level and whose corresponding resonance frequency has shifted $\sim 5 \mathrm{~Hz}$ [see the inset in Fig. 3(c)], provides clear evidence of this fact. When the probe is pushed against the sample, the shift in the TFs resonance frequency and the peak amplitude of the ultrasonic signal both increase simultaneously [dashed curve in Fig. 3(c)].

The arguments, given in the previous section, based on the viscosity $\eta$ to account for the dissipative effects as the probe gets closer to the sample are inadequate to explain the observed increase in the probe's resonance frequency. A conservative-type force needs to be identified. Although the electrical nature of the probe-specimen interaction has been favored before, ${ }^{8}$ the fading of the ultrasonic signal while the probe still remains immersed in the fluid layer (i.e., the charges are still there) makes this interpretation less likely in 
our experiment. A plausible alternative explanation is to consider the relatively slow viscoelastic response that the film might have in the regions very close to the surface. More precisely, we refer here to the viscosity effects described by the fluid's second viscosity coefficient $\zeta{ }^{26}$ the parameter relevant in processes accompanied by changes in density, i.e., processes accompanied by the generation of sound. A slight (right) lateral displacement of the probe would produce a more compressed region on the right and a less compressed region on the left if restoration of equilibrium does not happen fast enough. That is to say, a relatively slow dynamic response from the fluid gives rise to a net lateral restoring force, which is reflected in an increase of the probe's effective resonance frequency. Such an interpretation based on the rheological response of the film has been introduced before in the contexts of shear-force measurements, ${ }^{12}$ studies of molecular fluids confined between atomically flat surfaces, ${ }^{31}$ and ultrasound induced lubricity. ${ }^{32}$ The ultrasonic/shear-force microscope introduced in this article constitutes a new tool that allows for further advance and tests of these hypotheses, with the additional benefit of being able to contrast the results with the generation of sound. In this regard, additional experiments are underway to clarify probe-specimen interactions. ${ }^{33}$

${ }^{1}$ M. Ohtsu, K. Kobayashi, and Motoichi Ohtsu, Optical Near Fields: Introduction to Classical and Quantum Theories of Electromagnetic Phenomena at the Nanoscale (Springer, New York, 2004).

${ }^{2}$ M. A. Paesler and P. J. Moyer, Near-Field Optics: Theory, Instrumentation and Applications (Wiley, New York, 1996).

${ }^{3}$ F. Zenhausern, Y. Martin, and H. K. Wickramasinghe, Science 269, 1083 (1995).

${ }^{4}$ E. Betzig, P. L. Finn and J. S. Weiner, Appl. Phys. Lett. 60, 2484 (1992).

${ }^{5}$ R. Toledo-Crow, P. C. Yang, Y. Chen, and M. Vaez-Iravani, Appl. Phys. Lett. 60, 2957 (1992).

${ }^{6}$ K. Karrai and R. D. Grober, Appl. Phys. Lett. 66, 1842 (1995).

${ }^{7}$ K. Karrai and R. D. Grober, in Near Field Optics, SPIE Proceedings Series Vol. 2535, edited by M. A. Paesler and P. J. Moyer (SPIE, Bellingham, WA, 1995), pp. 69-81.

${ }^{8}$ M.-P. Bernal, F. Marquis Weible, P.-Y. Boillat, and P. Lambelet, Proc. IEEE 88, 1460 (2000).

${ }^{9}$ P. K. Wei and W. S. Fann, J. Appl. Phys. 87, 2561 (2000).

${ }^{10}$ M. J. Gregor, P. G. Blome, J. Schofer, and R. G. Ulbrich, Appl. Phys. Lett. 68, 307 (1996).

${ }^{11}$ K. Hsu and L. A. Gheber, Rev. Sci. Instrum. 70, 3609 (1999).

${ }^{12}$ C. L. Jahncke and H. D. Hallen, J. Appl. Phys. 93, 1274 (2003).

${ }^{13}$ K. Karrai and Ingo Tiemann, Phys. Rev. B 62, 13174 (2000).

${ }^{14} \mathrm{~B}$. Cavallier and B. Cretin, in Photoacoustic and Photothermal Phenomena: 10th International Conference, edited by F. Scudieri and M. Bertolotti, 1999, pp. 99-101.

${ }^{15}$ G. S. Shekhawat, G. A. D. Briggs, O. V. Kolosov, and R. E. Geer, in Characterization and Metrology for ULSI Technology: 2000 International
Conference, edited by D. G. Seiler, A. C. Diebold, T. J. Shaffner, R. McDonald, W. M. Bullis, P. J. Smith, and E. M. Secula, 2000, pp. 449-451.

${ }^{16}$ P. Lambelet, A. Sayah, M. Pfeffer, C. Philipona, and F. Marquis-Weible, Appl. Opt. 37, 7289 (1998).

${ }^{17}$ R. Stockle, C. Fokas, V. Deckert, and R. Zenobi, Appl. Phys. Lett. 75, 160 (1999).

${ }^{18}$ These values were estimated from the fitting of the experimental data [the "triangles" curve in Fig. 3(a)] to an RLC equivalent circuit of the TF, as described in Ref. 19. The series RLC resonator models the mechanical resonance of the tines, and a parallel capacitance $C_{p}$ gives the contacts and cables' capacitance. From this fitting the associated parameters are $C_{p}$ $=7.68 \times 10^{-12} \mathrm{~F}, L=7.35 \times 10^{3} \mathrm{H}, R=0.98 \times 10^{6} \Omega, C=3.22 \times 10^{-15} \mathrm{~F}$. To determine the mechanical oscillation amplitude, it is also necessary to find the piezoelectromechanical coupling constant $\alpha(\mathrm{C} / \mathrm{m})$, which can be determined from the expression $C=2 \alpha^{2} / K_{\text {stat }}$, where $K_{\text {stat }}$ is the spring constant of one of the TF's tines. The TF used here has dimensions $L$ $=3.8 \mathrm{~mm}, t=0.6 \mathrm{~mm}$, and $w=0.35 \mathrm{~mm}$, which gives $K_{\text {stat }}=(E / 4) w(t / L)^{3}$ $=26 \times 10^{3} \mathrm{~N} / \mathrm{m}$ and $\alpha=6.5 \times 10^{-6} \mathrm{C} / \mathrm{m}$. From the harmonic approximation motion $\Delta I=4 \pi f \alpha u_{0}$; at resonance the change in current is $\Delta I$ $=10 \mathrm{nA} \mathrm{rms}$, which gives $u_{0}=4 \mathrm{~nm} \mathrm{rms}$. This estimated value agrees well with the values reported in Ref. 7, where for a similar experimental setup as ours, for a $1 \mathrm{mV}$ driving voltage they observe $\Delta I=1 \mathrm{nA}$ and $0.5 \mathrm{~nm}$ amplitude. The equivalent driving force in our case turns out to be $F$ $=K_{\text {stat }} u_{0} / Q=60 \mathrm{nN}$.

${ }^{19}$ J. Rychen et al., Rev. Sci. Instrum. 71, 1695 (2000).

${ }^{20}$ G. He, M. H. Müser, and M. O. Robbins, Science 284, 1650 (1999).

${ }^{21}$ S. Davy, M. Spajer, and D. Courjon, Appl. Phys. Lett. 73, 2594 (1998).

${ }^{22}$ R. Brunner, O. Marti, and O. Hollricher, J. Appl. Phys. 86, 7100 (1999).

${ }^{23}$ Following Ref. 24, liquids that wet surfaces will spontaneously condense from the vapor into pores as bulk liquid. The radius of the water meniscus interface is related to the relative humidity of water $p / p_{\text {sat }}$ and the water surface tension $\gamma_{L}$ by the Kelvin's equation $r_{K}=\gamma_{L} V /\left[R T \log \left(p / p_{\text {sat }}\right)\right]$, where $V$ is the molar volume. $r_{K}$ ranges from 0.5 to $10 \mathrm{~nm}$ for values of $p / p_{\text {sat }}$ from 0.1 to 0.9 , respectively. Assuming the tip of the probe has a circular shape of radius $R$, the resulting attractive force pulling the probe and the (flat) surface together is calculated based on the pressure difference across the meniscus, $\Delta P=\gamma_{L} / r_{K}$, which acts on an effective area $\pi x^{2} \approx 4 \pi R r_{K}$. The attractive force for a tip of radius $R=50 \mathrm{~nm}$ is $\sim 45 \mathrm{nN}$.

${ }^{24} \mathrm{~J}$. Israelachvili, Intermolecular and Surface Forces, 2nd Ed. (Academic, New York, 1995).

${ }^{25}$ At $z=10 \mathrm{~nm}$ the extra horizontal restoring force can be expressed as $F_{x}$ $\sim 45 \mathrm{nN} \times \Delta x / z \sim(4.5 \mathrm{~N} / \mathrm{m}) \Delta x$; this additional value of $4.5 \mathrm{~N} / \mathrm{m}$ in the spring constant would shift the frequency by $\Delta f_{\text {res }}=\left(f_{\text {res }} \Delta k_{\text {stat }}\right) / 2 k_{\text {stat }}$ $\approx 3 \mathrm{~Hz}$, or greater for smaller $z$.

${ }^{26}$ L. D. Landau and E. M. Lifshitz, Fluid Mechanics, 2nd ed. (Pergamon, New York, 1987).

${ }^{27}$ The drag force on a sphere of radius $R=50 \mathrm{~nm}$ (as it moves with speed $v \sim 4 \times$ amplitude/period $=4 \times 4 \mathrm{~nm} \times 32680 \mathrm{~Hz}=5 \times 10^{-4} \mathrm{~m} / \mathrm{s}$, through liquid water of viscosity $\eta_{\text {bulk }}=10^{-3} \mathrm{Ns} / \mathrm{m}^{2}$ ), is given by $F_{\text {vis }}$ $=6 \pi \eta_{\text {bulk }} R v=5 \times 10^{-4} \mathrm{nM}$.

${ }^{28}$ S. Granick, Science 253, 1374 (1991).

${ }^{29}$ J. Klein and E. Kumacheva, J. Chem. Phys. 108, 6996 (1998).

${ }^{30}$ Y. Zhu and S. Granick, Phys. Rev. Lett. 93, 096101-1 (2004).

${ }^{31}$ A. Levent and S. Granick, J. Chem. Phys. 115, 1498 (2001).

${ }^{32}$ F. Dinelli, S. K. Biswas, G. A. D. Briggs, and O. V. Kolosov, Phys. Rev. B 61, 13995 (2000)

${ }^{33}$ X. Cui and A. H. La Rosa (unpublished). 
Review of Scientific Instruments is copyrighted by the American Institute of Physics (AIP). Redistribution of journal material is subject to the AIP online journal license and/or AIP copyright. For more information, see http://ojps.aip.org/rsio/rsicr.jsp 